\title{
Analysis on Nutritious Balance in Recipes Based on Diversity Indices and Cluster Analysis of Food Ingredients
}

\author{
Jiale Zhuang1, Junhui Gao² \\ ${ }^{1}$ Wuxi Big Bridge Academy, Wuxi, China \\ ${ }^{2}$ American and European International Study Center, Wuxi, China \\ Email: jhgao68@163.com
}

How to cite this paper: Zhuang, J.L. and Gao, J.H. (2018) Analysis on Nutritious Balance in Recipes Based on Diversity Indices and Cluster Analysis of Food Ingredients. Food and Nutrition Sciences, 9, 834-839.

https://doi.org/10.4236/fns.2018.97062

Received: June 18, 2018

Accepted: July 15, 2018

Published: July 18, 2018

Copyright $\odot 2018$ by authors and Scientific Research Publishing Inc. This work is licensed under the Creative Commons Attribution International License (CC BY 4.0).

http://creativecommons.org/licenses/by/4.0/

\begin{abstract}
This thesis research on nutritious balance of specific examples in Chinese recipes applying diversity indices and cluster analysis. Initially, based on data of the nutritional ingredients of food, such as proteins, fat and vitamins, we categorize 1200 kinds of specific food using cluster analysis; then, according to a recipe given by a local restaurant, we calculate and compare the diversity indices based on the components of 25 single dishes in the recipe and analyze the nutritious balance of each dish.
\end{abstract}

\section{Keywords}

Components of Food, Cluster Analysis, Recipe, Nutritious Balance, Diversity Indices

\section{Introduction}

The nutritional components of food include protein, fat, carbohydrates, calorie and minerals. All kinds of foods contain different components and proportions. People eat different kinds of food to satisfy the body's needs for various nutrients.

Liang Jia (2017) proposed that the human body needs a variety of nutrients to maintain the balance of the diet. The catering industry should pay attention to the dietary balance when providing food services for different consumer groups [1].

Zhang Fan, Guo Wenwei and Gao Zhihui (2017) also expressed that as the concept of healthy and green food widely spreads, the nutritious balance has become a problem that the catering industry must face in the process of promoting 
the service level and promoting the figure of its own. Therefore, for the catering industry, because of the different regions have different dietary habits, it is necessary to strengthen the scientific dietary combination according to the people of different consumer groups and of different constitutions [2].

Song Ce, Li Hao and Wu Libin (2017) study the proper intake of fruit based on the health of human body. First, the fruits are classified by systematic cluster analysis, according to the nutritional components. Then the annual per capita consumption of representative fruits is predicted with the trend moving average model, comparing the various nutrients needed to protect human health to evaluate the rationality of Chinese residents' annual intake level of minerals, vitamins, dietary fiber and other nutrients. Finally, a single objective linear programming model is established to calculate the annual reasonable per capita consumption of the main fruit products under the condition of nutritional balance to meet the health needs, so as to meet the nutritional health needs with a lower purchase cost [3].

In this paper, a new method for calculating the nutritional balance of recipes is proposed by combining cluster analysis with diversity indices.

\section{Materials and Methods}

\subsection{Data Sources}

Our original data mainly consists of food composition data and 25 dishes of food.

Food composition data from [4], containing more than 1200 records, including the sequence number, name, test ingredients, energy, water, protein, fat, vitamins, trace elements, and so on. Figure 1 shows 6 of them.

The 25 dishes of food are from the Sijinuantang restaurant in Wuxi. It contains double fresh with green pepper, original flavor chicken brine, broccoli with mashed garlic, tomato and egg soup and so on. Taking tomato and egg soup as an example, its food composition is shown in Table 1.

\subsection{The Principle or Concept of Cluster Analysis}

Cluster analysis refers to the grouping of physical or abstract objects into multiple classes consisting of similar objects. The goal of cluster analysis is to collect and classify data on similar basis. In different applications, many clustering techniques have been developed, which are used to describe data, to measure the

\begin{tabular}{|c|c|c|c|c|c|c|c|c|c|c|c|c|c|c|c|}
\hline Name & $\begin{array}{c}\text { Edible } \\
\text { part }\end{array}$ & Energy & Water & Protein & Fat & $\begin{array}{c}\text { Dietary } \\
\text { Fiber }\end{array}$ & Carbohydr & \begin{tabular}{|c|} 
Vitamin \\
$\mathrm{A}$ \\
\end{tabular} & $\begin{array}{c}\text { Vitamin } \\
\text { B1 } \\
\end{array}$ & $\begin{array}{c}\text { Vitamin } \\
\text { B2 }\end{array}$ & Niacin & $\begin{array}{c}\text { Vitamin } \\
\mathrm{E} \\
\end{array}$ & Sodium & Calcium & Iron \\
\hline millet & 100.0 & 349.0 & 11.3 & 13.6 & 2.7 & 3.5 & 67.6 & & 0.3 & 0.1 & 1.4 & 1.8 & 1.7 & 30.0 & 5.7 \\
\hline barley & 100.0 & 307.0 & 13.1 & 10.2 & 1.4 & 9.9 & 63.4 & & 0.1 & 0.1 & 5.0 & 0.3 & 1.6 & 13.0 & 5.1 \\
\hline paddy & 64.0 & 359.0 & 10.2 & 9.9 & 2.2 & 1.4 & 74.8 & & 0.1 & 0.1 & 5.0 & 0.3 & 1.6 & 13.0 & 5.1 \\
\hline rice & 100.0 & 346.0 & 13.3 & 7.4 & 0.8 & 0.7 & 77.2 & & 0.1 & 0.1 & 1.9 & 0.5 & 3.8 & 13.0 & 2.3 \\
\hline instant noodle & 100.0 & 472.0 & 3.6 & 9.5 & 21.1 & 0.7 & 60.9 & & 0.1 & 0.1 & 0.9 & 2.3 & 1144.0 & 25.0 & 4.1 \\
\hline bran & 100.0 & 220.0 & 14.5 & 15.8 & 4.0 & 31.3 & 30.1 & 20.0 & 0.3 & 0.3 & 12.5 & 4.5 & 12.2 & 206.0 & 9.9 \\
\hline
\end{tabular}

Figure 1. Food composition table (part). 
Table 1. Food Composition of tomato and egg soup.

\begin{tabular}{cccccc}
\hline Sub item & Mass(grams) & Type & Sub item & Mass(grams) & Type \\
\hline Tomato & 150 & Main Ingredient & Salt & 5 & Accessory \\
Egg & 100 & Main Ingredient & MSG & 2 & Accessory \\
Agaric & 50 & Main Ingredient & Sesame Oil & 1 & Accessory \\
Green Onion & 2 & Accessory & & & \\
\hline
\end{tabular}

similarity between different data sources, and to classify data sources into different clusters [5].

Chen Jian (2007) briefly introduced the concept and principle of cluster analysis [6]. Cluster analysis, also known as group analysis and point group analysis, is an ideal multivariate statistical technique for classification, mainly including partitioning clustering and hierarchical clustering. Partition clustering is also called segmentation clustering. Given a database of $\mathrm{M}$ data objects or tuples, the partition clustering is to construct $\mathrm{K}$ partition of data, each partition representing a cluster, and $\mathrm{k} \leq \mathrm{m}$. That is, it divides data into $\mathrm{K}$ groups and meets the following requirements: 1) each group contains at least one object; 2) each object must belong to and only belong to one group, while second requirements in some fuzzy partitioning techniques can be loosened. The hierarchical clustering divides the database into several levels, then divides and clusters the data of different levels, and outputs a hierarchical classification tree. Hierarchical methods can be divided into cohesive and divisive ones. The method of condensation, also known as a bottom-to-head approach, first takes each object as a cluster, and then merges a similar cluster into a growing cluster until all the objects are in a cluster, or a certain terminative condition is satisfied. The splitting method, also known as the head-to-bottom method, first places all the objects in a cluster and gradually subdivides them into smaller clusters, until each object becomes a cluster, or a certain terminative condition is satisfied.

This thesis uses K-MEANS method [7], which is one type of partitioning clustering. The idea of the method is: given the number of the classes $\mathrm{K}$, divide $\mathrm{N}$ objects into $\mathrm{K}$ classes, making the similarity among the objects in the class the most, and the similarity among the classes is the smallest. The calculation of similarity is carried out according to the average value of the objects in a cluster (seen as the center of clustering); that is, each cluster is represented by the average value of the objects in the cluster. The greatest advantage of the method is simplicity and efficiency. The key of the method is the initial center selection and the formula for calculation of the similarity among classes.

\subsection{How to Implement Clustering Analysis}

We used Python3.6 and sklearn.cluster's KMeans to cluster analysis, and carried out 20 types and 30 types of cluster analysis respectively. Sklearn is a commonly used Python third party module in machine learning, which can be installed through pip. 


\subsection{Diversity Induces}

The Shannon Wiener diversity index [8] is an index for investigating local diversity in plant communities (alpha diversity), often used in conjunction with the Simpson diversity index. The Shannon Wiener index formula is:

$$
H=-\sum(P i)(\ln P i)
$$

ln was also replaced by $\log 2, P i$ for the proportion of individual species in the total number of individuals. In this formula, $H=$ the information content of samples $=$ the diversity index of the community, $S=$ species number, $P i=$ the proportion of the individuals belonging to the $i$ species in the sample. If the total number of samples of the sample is $N$, and the number of i species is $n i$, then $P i=n i l N$.

In the Shannon Wiener index, there are two components: species number and distribution uniformity among individuals (equability or evenness). The more evenly distributed individuals are, the greater the $\mathrm{H}$ value. If each individual belonged to different species, the diversity index would be the largest; if each individual belonged to the same species, the diversity index would be the smallest.

\subsection{Calculation of Nutritious Balance}

We use formula (1) to calculate the nutritional balance. In the formula, Pi refers to the total mass of each dish in group $i$ and the percentage of the total mass of all ingredients. A total of 20 groups are generated by clustering.

Take salad ribs as an example. The mass percentages of species number 3, 0 , 17 , and 19 were $0.9597,0.0192,0.0154,0.0058$ respectively. The equilibrium index calculated by formula (1) was 0.3017 .

In actual calculation, the quality data (content) of each component of the dish is given by the chef of Wuxi Four Seasons restaurant.

\section{Results}

\subsection{Clustering Results in the Food List}

Observing the 20 species, the distribution of 1200 foods in 20 species is shown in the Table 2.

\subsection{Clustering Information of Twenty-Five Dishes}

Taking tomato egg soup as an example, on the basis of Table 2, ID (identification number of more than 1200 foods) of the subitems, species number of 20 clusters and species number of 30 clusters were added. For example, in Chart 3 the species number of 20 clusters of eggs is 3 .

From Table 3, we can see that the subterm salt and monosodium glutamate of the tomato and egg soup belong to the 17 category of the 20 classes, and the quality is added when the formula (1) is used to calculate the nutritional balance.

\subsection{Comparison of Nutritious Balance between Twenty-Five Dishes}

Using formula (1) and the method mentioned in the preceding 2.5 sections, the 
data of nutrient balance of 25 dishes are calculated, as shown in Table 4.

\section{Discussion}

We clustered basic foods into 20 categories, and then analyzed and calculated the nutritional balance of vegetables. Well, is the 20 category reasonable? In fact, we have clustered in 30 categories. The results are different from those of 20 categories, but there is little difference. From Table 3, we found that there are 20

Table 2. Distribution and examples of various kinds of food.

\begin{tabular}{|c|c|c|c|c|c|}
\hline Species Number & Number of Food & Examples & Species Number & Number of Food & Examples \\
\hline 0 & 163 & Cabbage, Pear & 10 & 260 & Peach, Radish \\
\hline 1 & 70 & Chicken & 11 & 273 & Rice, Flour \\
\hline 2 & 3 & Ox liver & 12 & 6 & Gomphidius rutilus \\
\hline 3 & 164 & Egg, Intestine & 13 & 1 & Sweet pepper \\
\hline 4 & 139 & Walnut, Fish & 14 & 9 & Yolk \\
\hline 5 & 1 & Starch & 15 & 3 & Mushroom \\
\hline 6 & 10 & Sesame & 16 & 2 & Russula Sanguinaria \\
\hline 7 & 23 & Milk powder & 17 & 2 & Salt \\
\hline 9 & 29 & Salad oil & 19 & 48 & Soy sauce \\
\hline
\end{tabular}

Table 3. Cluster information of tomato and egg soup.

\begin{tabular}{|c|c|c|c|c|c|}
\hline Subitem & Mass (grams) & Type & ID & 20 clusters & 30 clusters \\
\hline Tomato & 150 & Main Ingredient & 937 & 10 & 29 \\
\hline Agaric & 50 & Main Ingredient & 238 & 18 & 17 \\
\hline Green Onion & 2 & Accessory & 891 & 0 & 7 \\
\hline Salt & 5 & Accessory & 1208 & 17 & 16 \\
\hline MSG & 2 & Accessory & 1207 & 17 & 16 \\
\hline \multirow[t]{2}{*}{ Sesame Oil } & 1 & Accessory & 1109 & 9 & 19 \\
\hline & & & & 6 species & 6 species \\
\hline
\end{tabular}

Table 4. Comparison of nutritious balance between 25 dishes.

\begin{tabular}{cccccc}
\hline & Index & Name & Index & Name & Index \\
\hline Name & 2.472 & Field flavors & 1.836 & Stinky mandarin fish & 1.506 \\
Assorted gluten & 2.367 & Celery and yam and black fungus & 1.713 & fried whole fish that looks like a squirrel & 1.444 \\
Fat beef in sour soup & 2.191 & Fresh shellfish with nuts and mushrooms & 1.701 & baby cabbage in chicken soup & 1.373 \\
Fish with Japanese pepper & 2.145 & Tomato and egg soup & 1.655 & mushroom with Goose liver sauce & 1.333 \\
ring oil eel paste & 1.920 & Black tofu & 1.630 & Fresh shellfish with egg white & 1.329 \\
\hline
\end{tabular}


and 30 two species of clustering, two subitems of salt and monosodium glutamate, which belong to seventeenth in 20 of the 20 , and all belong to the 16 class at the time of 30 , and still belong to the same category. In addition, after two clustering methods, the number of subitems is 6 . This shows that clustering is changed from 20 to 30 , and the result is stable.

In general, the distance between different centers is not equal, which will affect the accuracy of nutrition balance calculation.

The deficiency of this article is that the food ingredients and recipes in China are analyzed only, but not in other countries.

\section{Conclusions}

In this paper, a new method for calculating the nutritional balance of recipes is proposed by combining cluster analysis with diversity index for the first time. In this paper, 25 Chinese dishes were calculated and compared. Among them, green pepper had the highest nutrition balance index, while the salad had the lowest nutrition balance index.

This method can be applied to food composition and menu analysis in all countries, so it is easy to popularize.

\section{Acknowledgements}

The chef of Wuxi four seasons warm hall restaurant provides us with the detailed data of 25 dishes, including the composition of each dish, the quality of the component, and thank you here.

\section{References}

[1] Liang, J. (2017) Principles and Necessities of Balanced Diet and Nutrition in Catering Industry. Food Safety Weekly, No. 12, 57-57.

[2] Zhang, F., Guo, W.W. and Gao, Z.H. (2017) Principles and Necessity of Balanced Diet and Nutrition in Catering Industry. Consumer Guide, No. 26.

[3] Song, C., Li, H. and Wu, L.B. (2017) Analysis of Reasonable Intake of Fruit Based on Nutritional Health Perspective. Journal of Qiqihar University (Natural Science), 4.

[4] Wang, G.Y. (2009) Chinese Food Composition List. The Medical Press of Peking University, Beijing.

[5] https://baike.baidu.com/item/\%E8\%81\%9A\%E7\%B1\%BB\%E5\%88\%86\%E6\%9E\%90 /3450227?fr=aladdin

[6] Chen, J. (2007) Analysis of Common Cluster Analysis Algorithms. Journal of Anhui Electronic Information Vocational and Technical College, 1, No. 6.

[7] MacQueen, J. (1967) Some Methods for Classification and Analysis of Multivariate Observations. Proceedings of the Fifth Berkeley Symposium on Mathematical Statistics and Probability, 1, 281-297.

[8] Pielou, E.C. (1975) Ecological Diversity. Wiley \& Sons, New York. 